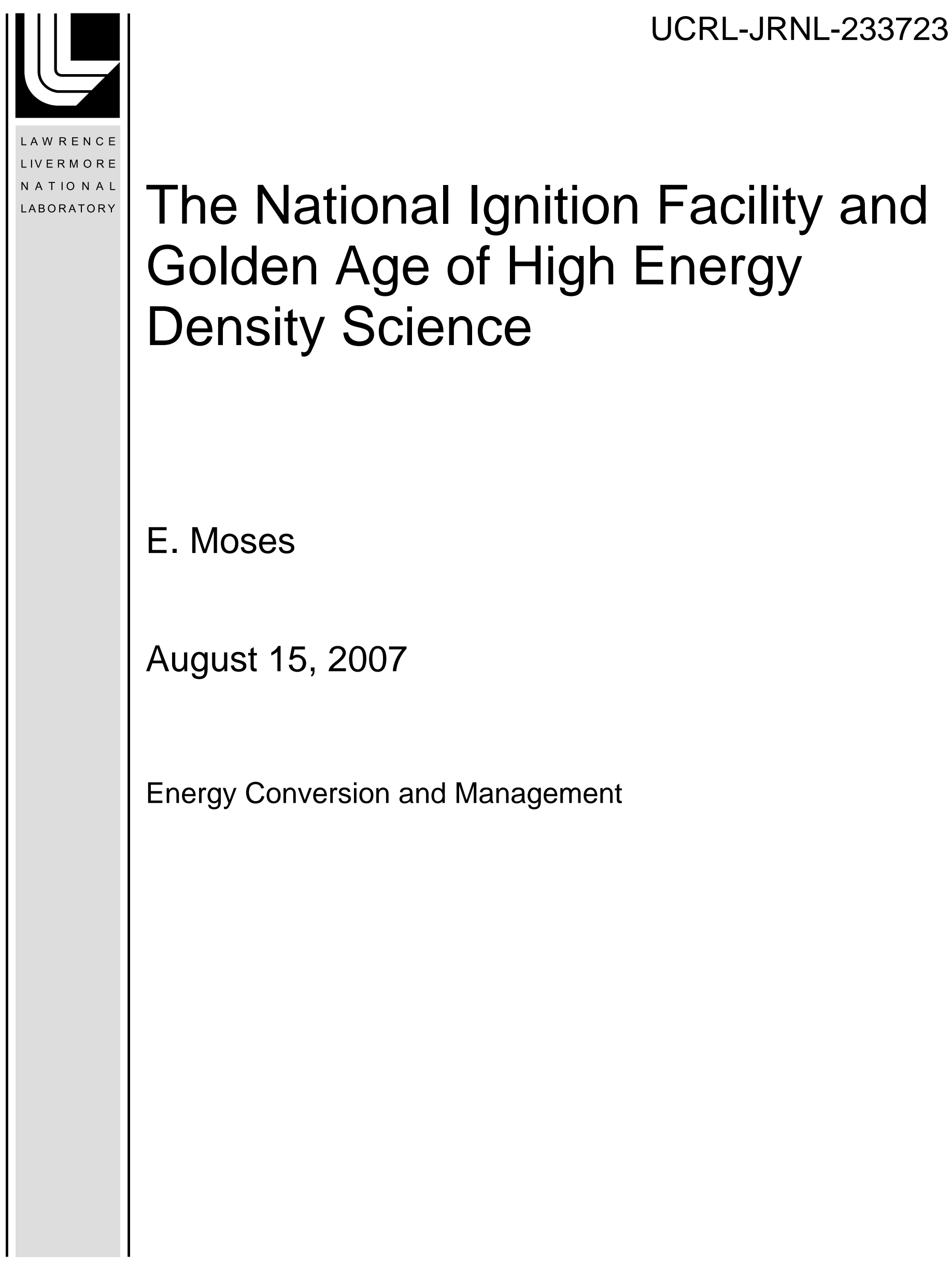


This document was prepared as an account of work sponsored by an agency of the United States government. Neither the United States government nor Lawrence Livermore National Security, LLC, nor any of their employees makes any warranty, expressed or implied, or assumes any legal liability or responsibility for the accuracy, completeness, or usefulness of any information, apparatus, product, or process disclosed, or represents that its use would not infringe privately owned rights. Reference herein to any specific commercial product, process, or service by trade name, trademark, manufacturer, or otherwise does not necessarily constitute or imply its endorsement, recommendation, or favoring by the United States government or Lawrence Livermore National Security, LLC. The views and opinions of authors expressed herein do not necessarily state or reflect those of the United States government or Lawrence Livermore National Security, LLC, and shall not be used for advertising or product endorsement purposes. 


\title{
THE NATIONAL IGNITION FACILITY AND THE GOLDEN AGE OF HIGH ENERGY DENSITY SCIENCE
}

\author{
Edward I. Moses
}

Lawrence Livermore National Laboratory

Livermore, CA 94550 


\section{ABSTRACT}

The National Ignition Facility (NIF) is a 192-beam $\mathrm{Nd}$ :glass laser facility being constructed at the Lawrence Livermore National Laboratory (LLNL) to conduct research in inertial confinement fusion (ICF) and high energy density (HED) science. When completed, NIF will produce $1.8 \mathrm{MJ}, 500 \mathrm{TW}$ of ultraviolet light, making it the world's largest and highest-energy laser system. The NIF is poised to become the world's preeminent facility for conducting ICF and fusion energy research and for studying matter at extreme densities and temperatures.

\section{INTRODUCTION}

Construction of the NIF project, which began in 1996 , is currently over $90 \%$ complete and scheduled for completion in 2009. Half of the 192 main laser beams have been fully commissioned and are operational. Installation and commissioning of the remaining optics and electronics will be completed over the next year and a half, leading to the beginning of ignition experiments in 2010.

The NIF will provide opportunities to explore regimes of high energy density physics never before possible. In concert with other national facilities such as $\mathrm{Z}$ at Sandia National Laboratories and OMEGA at the University of Rochester and with several university laboratories, the U.S. will have unique opportunities to expand the forefront of science in areas that will safeguard our national security, enhance our ability to understand our universe, and provide alternatives for clean energy in the future.

\section{THE NATIONAL IGNITION FACILITY}

\section{A. The Laser}

The NIF laser is comprised of 192 laser beams, each delivering over $20 \mathrm{~kJ}$ of optical energy at $1053 \mathrm{~nm}$. The Nd:glass NIF laser utilizes a multi-pass laser architecture to achieve a total system gain of greater than $10^{15}$ (Figure 1).

The NIF laser starts as a single beam generated by the master oscillator, a highly stable fiber oscillator with the capability of generating arbitrary optical pulse shapes. This single beam is amplified and split into 48 separate beams to drive the injection laser system.
The injection laser system, one of the most challenging and sophisticated laser amplifiers on NIF, amplifies the nanojoule optical pulses from the master oscillator by a factor of over $10^{10}$. The several hundredmillijoule output optical pulses from the injection laser system are then split into four separate beams and injected into the main laser beam lines at the transport spatial filter.

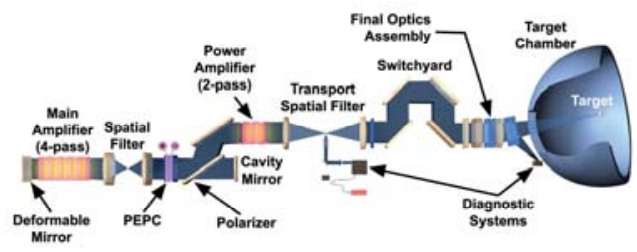

Figure 1. Simplified diagram of NIF laser architecture.

Once in the main laser, the individual beams are expanded to $40 \times 40 \mathrm{~cm}$ and amplified to over $20 \mathrm{~kJ}$ through a multi-pass configuration. This multi-pass configuration is implemented utilizing a large-aperture plasma electrode Pockels cell (PEPC) to switch the optical pulses through the amplifier system. After four passes through the main amplifier and two passes through the power amplifier, the beam is directed to the target chamber through a system of optics in the switchyards.

All laser, target and diagnostic systems are controlled from a single control room through an advanced and complex distributed control system. Over 60,000 control points are carefully orchestrated with over 1.5 million lines of code to precisely execute each shot and experiment on NIF.

At the target chamber (Figure 2), the beam enters the final optics assemblies where it is converted to ultraviolet at $351 \mathrm{~nm}$ and focused on the centimetersize target suspended in the center of the target chamber. A large array of sophisticated diagnostics will probe the laser-target interactions and provide quality data for researchers. 


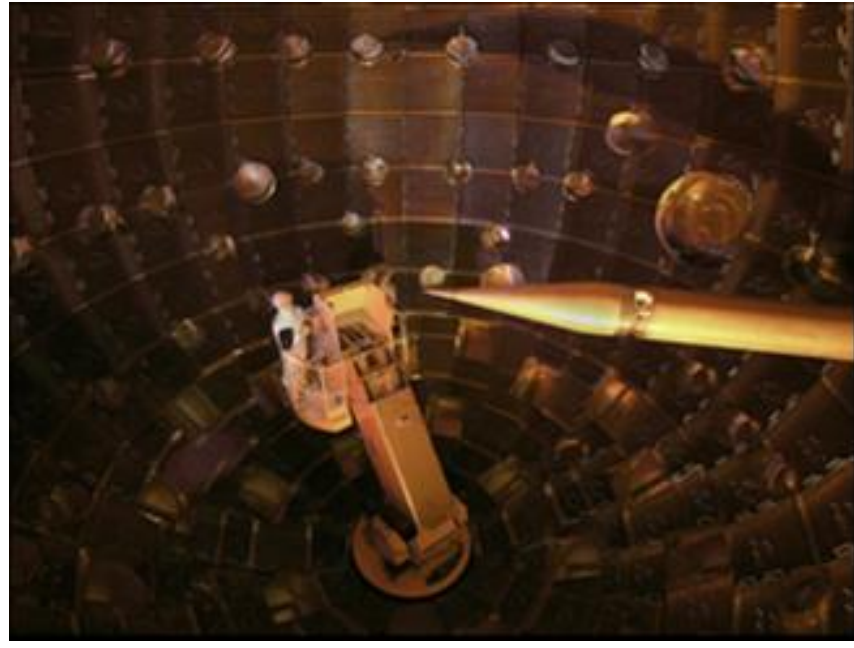

Figure 2. The NIF target chamber is constructed from 4-inch-thick aluminum and coated with a 16-inchthick shielding concrete shell. The entire assembly weighs about one million pounds. The target positioner, which holds the target at its tip, is on the right. The target chamber service system can be manned by two operators

\section{B. Targets}

All experiments on NIF have one common requirement: illuminating a sub-centimeter scale target, precisely centered in the target chamber. Creating a NIF target is a complex interplay among target designers, materials scientists, and engineers. Designers understand the goals for each experiment and must establish specifications for the target. NIF targets are typically only a few millimeters in size, and their complicated shapes must be machined to meet precise requirements, including specifications for density, concentricity, and surface smoothness.

Targets for the ICF experiments are being fine tuned by a large collaboration that includes scientists and engineers from LLNL, General Atomics in San Diego, the University of Rochester's Laboratory for Laser Energetics, Los Alamos National Laboratory (LANL) and other industrial partners. This team is perfecting the target materials and methods to fabricate them. Nanoscale materials developed for NIF experiments include high-density carbon, very low-density copper and gold foams, and graded-density foams.

Manufacturing requirements for all NIF targets are extremely challenging. Components must be machined to within an accuracy of $1 \mu \mathrm{m}$, or one-millionth of a meter. Joints can be as small as $100 \mathrm{~nm}$, which is just one-thousandth the width of a human hair. In addition, the margin of error for target assembly is less than $8 \mu \mathrm{m}$. The extreme temperatures and pressures the targets will encounter during experiments make the results highly susceptible to imperfections in fabrication.
The current design for the ICF target is a copper-doped beryllium capsule with a smooth solid layer of the hydrogen isotopes, deuterium and tritium (D-T), on its inner surface. The radially tailored capsule is made of a lightweight material (Figure 3) and fits inside a 9-mmhigh by 5-mm-wide hohlraum cylinder made of a material with a high atomic number such as gold (Figure 4).

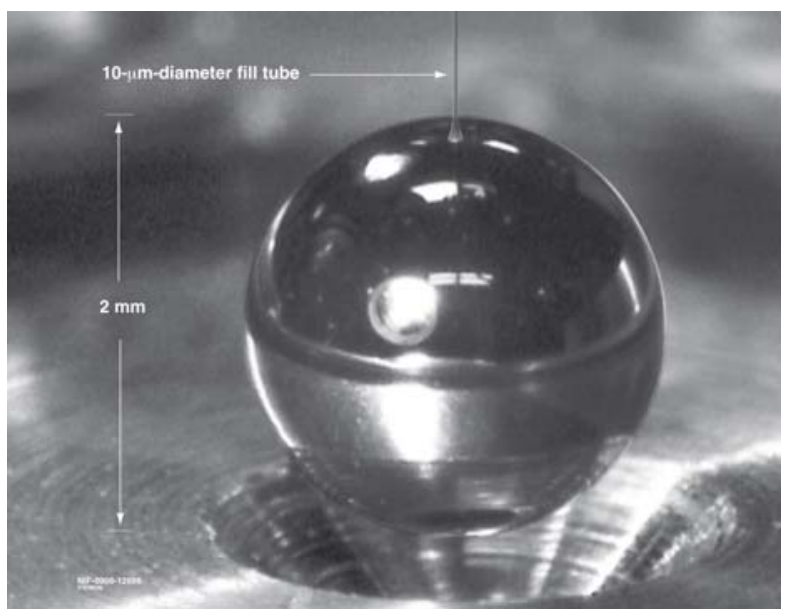

Figure 3. Beryllium capsule and fill tube.

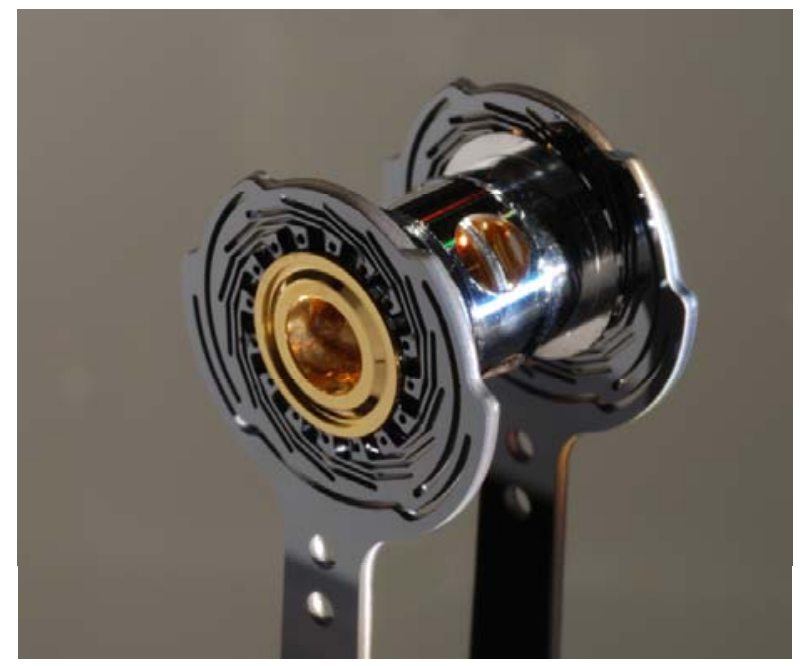

Figure 4. NIF target assembly.

When NIF's laser beams impinge on the hohlraum's inner cavity, laser energy is converted to X-ray energy. These X-rays bathe the capsule and ablate its outer layer. Conservation of momentum requires that the remaining material implode or compress. Compressing the D-T fuel to extraordinarily high temperature, pressure, and density ignites a burning hydrogen plasma. 
For NIF to achieve ignition, the beryllium capsule must have a precise spherical shape. The capsule's surfaces must be smooth to within $1 \mathrm{~nm}$-an unprecedented requirement for surface roughness - and the thickness and opacity of the copper-doped layers must be carefully controlled.

\section{III.CAMPAIGN TO IGNITION}

A detailed plan called the National Ignition Campaign (NIC) has been developed to begin ignition experiments in 2010. NIC is a collaborative effort involving LLNL, the University of Rochester Laboratory for Laser Energetics, General Atomics, LANL, and Sandia National Laboratories (SNL). Components of the NIC plan include target physics, systems engineering and operations, target fabrication, and equipment development such as target diagnostics, cryogenic target positioner, and user optics required for the ignition experiment. Target designs have been developed that calculate to ignite at energy of approximately $1 \mathrm{MJ}$.

Experiments using the OMEGA laser at the University of Rochester are helping to validate these designs. Development of manufacturing capability is well under way for producing targets to the required tolerances. Diagnostics and other support equipment are being designed and fabricated to help perform the ignition experiments.

The NIF Project and NIC activities are merging at a rapid rate. The NIF laser and the equipment needed for ignition experiments, including high-quality targets, will be available in March 2009. In less than a year we will be commissioning laser beams to the target chamber. We have planned an early campaign with 96 beams, called Eos, which will allow us to choose the optimum hohlraum temperature and laser energy for initial ignition experiments. Eos, the Greek goddess of the dawn, is a fitting name because NIF represents the dawn of a new era in physics research, especially in the field of HED science.
The range of operations where Laser Plasma Interaction (LPI) is manageable in the ignition-point design is bounded by hohlraum temperatures of 270$300 \mathrm{eV}$. Starting with the Eos series, NIF will be established as a preeminent international HED physics facility, which will set the facility directly on the path to the first ignition experiments in 2010. The initial ignition experiments will only scratch the surface of NIF's potential, which includes high yields with green light and greatly expanded opportunities for the uses of ignition by decoupling compression and ignition with the Fast Ignition (FI) approach.

\section{IV.EXPLORING THE FRONTIERS OF SCIENCE}

In addition to supporting the DOE's Stockpile Stewardship Program, NIF will provide researchers from universities and DOE national laboratories unparalleled opportunities to explore basic science in astrophysics, planetary physics, hydrodynamics, nonlinear optical physics and materials science. Approximately $15 \%$ of NIF facility time and a larger percentage of its shots will be devoted to science experiments in these fields. The first science studies will focus on recreating in the laboratory the properties of celestial objects under scaled conditions. With its 192 beams together generating up to $1.8 \mathrm{MJ}$ of ultraviolet energy, the giant laser will allow scientists to explore some of the most extreme conditions in the universe such as the hot, dense plasmas found in stars. NIF experiments will help scientists understand the mechanisms driving new stars, supernovae, black holes and the interiors of giant planets. The physical processes of stars have long been of interest to LLNL researchers because the prime stellar energy mechanism, thermonuclear, is central to the Laboratory's central mission. For decades, researchers have advanced astrophysics by applying their expertise in HED physics and computer modeling of the nuclear processes that take place in these regimes.

Once NIF attains ignition (a burst of fusion reactions in which more energy is liberated than is input), a flux of $10^{32}$ to $10^{33}$ neutrons per square centimeter per second will be generated, a rate that may allow excitedstate nuclear reactions to occur. This neutron flux will also enable scientists to extend their understanding of the nucleosynthesis of heavy elements, those nuclei more massive than iron. Scaled NIF experiments will permit studies of the entire life cycle of a star, from its birth in a cold, dense molecular cloud through its subsequent stages of evolution to an explosive death such as a supernova. 
Once formed, stars are heated by nuclear fusion in the interior and cooled by radiation emissions at their surface, called the photosphere. Opacities of each layer control the rate at which heat moves from the core to the surface. In this way, opacity plays a major role in understanding the evolution, luminosity, and instabilities of stars. Experiments will mimic stellar plasma to obtain information on the opacities of key elements such as iron and determine how opacity changes with plasma density and temperature throughout a star's lifetime. Experimenters plan to simultaneously measure the radiation transmission, temperature, and density of a material sample.

\section{CONCLUSIONS}

NIF represents bold and courageous thinking. All prior large laser facilities were designed and built with the latest technologies, and scientists then determined what research the facility could accomplish. In contrast, NIF was designed specifically to meet the needs of three missions: strengthening stockpile stewardship for a safe and reliable nuclear stockpile without nuclear testing, advancing ICF as a clean source of energy, and making significant strides in HED physics.

These three missions share the need to expose materials to extraordinarily high pressures, temperatures, and densities_as much as 100 billion atmospheres pressure, 100 million degrees Centigrade temperature, and $1,000 \mathrm{~g} / \mathrm{cm}^{3}$ density. These conditions occur in exploding nuclear weapons, in supernovae, and in the fusion reactions that power our Sun and other stars and that may one day provide an inexhaustible power supply on Earth. Because of the similarities of these phenomena, the results of some NIF experiments will be applicable to all three missions.

We cannot venture inside stars, planets, or black holes, nor can we traverse billions of light years across the universe to examine a supernova explosion. However, with NIF, we can re-create, on a vastly smaller scale, the same physical processes that astronomers can only glimpse through a telescope. 2010 marks the golden anniversary of the demonstration of the first laser and the concept of ICF. Our goal with the National Ignition Campaign is to demonstrate ignition and burn, in turn launching a new era of high energy density science and energy research.

Work performed under the auspices of the U.S. Department of Energy by the University of California, Lawrence Livermore National Laboratory under Contract No. W-7405-ENG-48. 